\title{
Roles of the Clr4 methyltransferase complex in nucleation, spreading and maintenance of heterochromatin
}

\author{
Ke Zhang ${ }^{1}$, Kerstin Mosch $^{2}$, Wolfgang Fischle ${ }^{2}$ \& Shiv I S Grewal ${ }^{1}$
}

\begin{abstract}
Heterochromatin assembly, involving methylation of histone $\mathrm{H} 3$ lysine 9 (H3K9me), regulates various chromosomal processes. In fission yeast, heterochromatin targeted to specific repeat loci in an RNAi-dependent manner spreads across extended domains to exert regional epigenetic control. The $\mathrm{Clr} 4$ methyltransferase complex $(\mathrm{ClrC})$ is responsible for nucleation and spreading of heterochromatin; however, its recruitment to heterochromatic repeats is poorly understood. Here we demonstrate that CIrC components are distributed throughout heterochromatic domains. To nucleate heterochromatin, Rik1, a WD domaincontaining subunit of $\mathrm{ClrC}$, is loaded onto the transcribed repeats via RNAi machinery including the RNA-induced transcriptional silencing (RITS) complex. Furthermore, we show that the chromodomain of Clr4 binds specifically to $\mathrm{H} 3 \mathrm{~K} 9 \mathrm{me}$ that is essential for the spreading of heterochromatin. Our analyses delineate sequential steps for the assembly of heterochromatic domains and suggest that the ability of Clr4 to both 'write' and 'read' H3K9me facilitates heterochromatin maintenance through successive cell divisions.
\end{abstract}

Higher-order chromatin structures that underlie almost all aspects of chromosome behavior are essential for the functional organization of eukaryotic genomes. The formation of heterochromatin at developmentally regulated loci and at repetitive DNA elements such as transposons has a crucial role in epigenetic gene control, suppression of recombination and proper segregation of chromosomes during cell division $^{1-3}$. Heterochromatin has the unique abilities to spread and to serve as a multipurpose platform for the recruitment of diverse regulatory proteins, thereby influencing gene expression and other chromosomal processes in a region-specific, sequence-independent manner ${ }^{2,4}$. Heterochromatic structures are recapitulated during chromosome replication and seem to be inherited through cell division in a clonal, metastable fashion ${ }^{5-8}$. The mechanisms facilitating the inheritance of heterochromatic structures are not fully understood. Misregulation of such processes is believed to cause developmental defects and might contribute to cellular transformations ${ }^{9}$.

In the fission yeast Schizosaccharomyces pombe, the heterochromatic mark $\mathrm{H} 3 \mathrm{~K} 9 \mathrm{me}$ and its interacting protein Swi6 (a HP1 family protein) are localized to large chromosomal domains at the silent mating-type (mat) locus, the pericentromeric regions and the subtelomeres ${ }^{2,10}$. These loci contain a specific class of repeat elements $(d g$ and $d h$ ) that serve as RNAi-dependent heterochromatin nucleation centers ${ }^{6,10,11}$. RNA polymerase II (RNAPII) transcription of repeat elements generates transcripts that are converted into small interfering RNAs (siRNAs) by RNA interference (RNAi) machinery including
Argonaute (Ago1), RNA-dependent RNA polymerase (Rdp1) and Dicer (Dcr1 $)^{10,12-17}$. siRNAs bind to the RITS complex, which consists of Ago1, Tas3 and the chromodomain protein Chp1, to facilitate the loading of RNAi and heterochromatin factors ${ }^{18}$. This process, which is coupled to preferential RNAPII transcription of repeat elements during $\mathrm{S}$ phase ${ }^{19}$, directs $\mathrm{H} 3 \mathrm{~K} 9$ me to the targeted chromatin regions. $\mathrm{H} 3 \mathrm{~K} 9 \mathrm{me}$ then provides binding sites for the loading of Swi6 and Chp2, two chromodomain proteins essential for the assembly of heterochromatic structures ${ }^{10,20-22}$. H3K9me further allows RITS to stably associate with heterochromatic regions via the chromodomain of Chp1 (ref. 23). In the current model, a selfreinforcing loop mechanism in which chromatin-bound RITS recruits factors such as Rdp1 enables the RNAi machinery to act as a stable component of heterochromatin that processes repeat transcripts into siRNAs, which are required for further stabilization of heterochromatin ${ }^{15,23}$.

Studies at the $S$. pombe mat locus have shown that heterochromatin nucleated at specific sites-either via the RNAi machinery acting through the $d g$ - and $d h$-like element $c e n H$ or via DNA binding factors bound to silencer DNA elements-spreads across extended domains ${ }^{2,6,23,24}$. Spreading of heterochromatin is essential for various chromosomal processes including long-range chromatin interactions and silencing of developmentally regulated mat $\operatorname{loci}^{2}$. Once assembled, the heterochromatic state is inherited in cis during mitosis, and remarkably even through meiosis, as a Mendelian-like marker ${ }^{6,7}$. It

${ }^{1}$ Laboratory of Biochemistry and Molecular Biology, National Cancer Institute, National Institutes of Health, Bethesda, Maryland 20892, USA. ${ }^{2}$ Laboratory of Chromatin Biochemistry, Max Planck Institute for Biophysical Chemistry, Am Fassberg 11, 37077 Göttingen, Germany. Correspondence should be addressed to S.I.S.G. (grewals@mail.nih.gov).

Received 8 January; accepted 22 February; published online 16 March 2008; doi:10.1038/nsmb.1406 
is believed that the persistence of macromolecular protein complexes associated with the locus contributes to the maintenance of heterochromatic structures ${ }^{6-8}$.

Post-translational modifications of histones and other factors facilitate the maintenance of heterochromatin. In S. pombe, histone deacetylases such as Sir2, Clr6 and Clr3 of the SHREC (Snf2-HDAC repressor complex) contribute to heterochromatin assembly ${ }^{25-29}$. Another key factor is $\mathrm{Clr} 4$, an H3K9 methyltransferase that shares structural and functional similarities to mammalian Suv39h $\mathrm{h}^{30,31}$. Clr4 contains an N-terminal chromodomain and a C-terminal SET catalytic core domain ${ }^{32}$. Recent evidence suggests that $\mathrm{Clr} 4$ is a component of a multisubunit protein complex, ClrC, containing Cul4, Rik1, Raf1 and Raf2 proteins ${ }^{3-36}$. These factors along with a ring-box protein (Rbx1) are components of an E3 ubiquitin ligase, whose activity is essential for heterochromatin assembly ${ }^{33,35}$. Cul4 belongs to the cullin protein family that serves as a scaffold for the assembly of ubiquitin ligases. Rik1, which contains several WD repeats, shares similarity with nucleic acid binding proteins such as the damaged DNA binding protein DDB1 and the cleavage polyadenylation factor CPSF-A ${ }^{37}$. Although Raf1 also contains WD repeats, Raf2 shares no obvious sequence similarity with other proteins ${ }^{33,34,36,38}$. Loss of Clr4-associated factors affects $\mathrm{H} 3 \mathrm{~K} 9 \mathrm{me}^{33,34,36,38}$, but the mechanistic contributions of the different components of the ClrC complex remain to be explored.

In this study, we define the sequential steps of $\mathrm{ClrC}$ recruitment that are important for the assembly and propagation of heterochromatic structures. We show that, to nucleate heterochromatin, Rik1 is targeted specifically to RNAPII-transcribed centromeric repeat elements in an RNAi-dependent manner. Our analyses further uncover an important functionality of the Clr4 methyltransferase, which is to both write and read the H3K9me chromatin mark. We show that $\mathrm{Clr} 4$ binds specifically to $\mathrm{H} 3 \mathrm{~K} 9 \mathrm{me}$ via its chromodomain. This interaction facilitates the spreading and stable maintenance of heterochromatic structures during cell division, thereby providing a mechanism for the epigenetic inheritance of a defined chromatin state.

\section{RESULTS}

\section{CIrC localizes throughout heterochromatic domains}

To gain further insights into the roles of $\mathrm{ClrC}$ subunits in heterochromatin assembly, we mapped their genomic distributions by performing chromatin immunoprecipitation (ChIP) coupled with DNA microarray analysis (ChIP-chip). These experiments showed Clr4, Raf1, Raf2, Rik1 and Cul4 enrichments at major heterochromatic loci such as preicentromeric regions, subtelomeres, ribosomal DNA
(rDNA) and the silent mat locus (Fig. 1 and Supplementary Fig. 1 online), consistent with the involvement of $\mathrm{ClrC}$ in $\mathrm{H} 3 \mathrm{~K} 9 \mathrm{me}$ at these loci ${ }^{10}$. Furthermore, we detected low levels of $\mathrm{ClrC}$ binding at several euchromatic sites, including noncoding RNAs, intergenic regions and meiotic genes (Fig. 1). The binding of $\mathrm{ClrC}$ components at these loci is reminiscent of the localization of heterochromatin factors at euchromatic loci in the genomes of higher eukaryotes ${ }^{1,39,40}$ and may suggest a potential involvement of $\mathrm{ClrC}$ in modifying chromatin at these loci. We noticed that some $\mathrm{ClrC}$ components localize to several sites, seemingly unaccompanied by other components (Fig. 1). This observation might reflect the dynamic nature of $\mathrm{ClrC}$ or indicate that these factors are recruited independently in certain chromosomal 


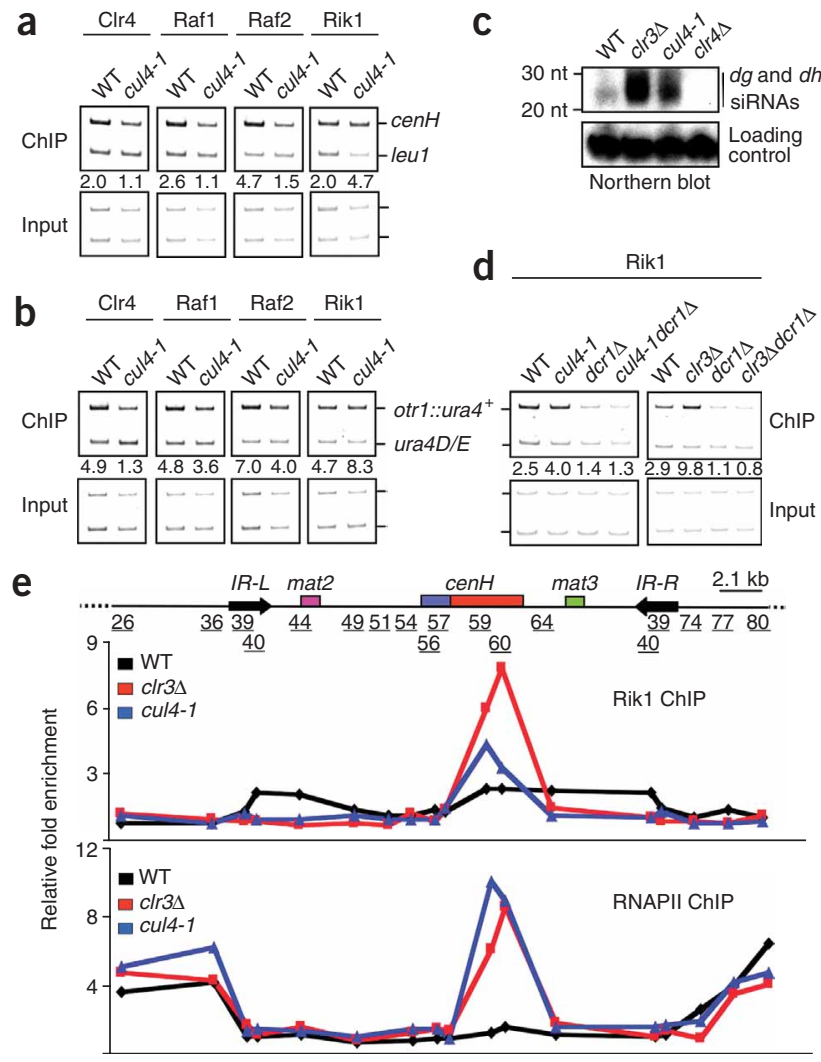

contexts. The enrichment levels of Cul4, which shows broad chromosomal distribution consistent with its roles in various cellular pathways $^{41}$, were relatively low compared to the levels of other $\mathrm{ClrC}$ subunits. This result may reflect either lower occupancy or a more peripheral chromatin association of Cul4 that is not efficiently detected by ChIP. In any case, Cul4 could be reproducibly detected at heterochromatic loci (Supplementary Fig. 2 online).

Detailed analysis showed that, at centromeres, high levels of $\mathrm{ClrC}$ were confined to the outer repeat (otr) region, which contain $d g$ and $d h$ repeats as well as a portion of the innermost repeats (imr). On both sides of these heterochromatic domains, there was a sharp decrease in ClrC localization (Supplementary Fig. 1a) that coincided with the previously described boundary elements ${ }^{2}$. At the mat locus, all subunits of ClrC were preferentially enriched across a 20 -kb heterochromatic domain surrounded by the inverted repeat (IR) boundaries (Supplementary Fig. 1b). A similarly broad distribution of Clr4, Raf1, Raf2, Rik1 and Cul4 was also observed across a $\sim 40$-kb domain at the subtelomeric regions (Supplementary Fig. 1c). In addition, we observed substantial enrichment of ClrC subunits, in particular Rik1 and Raf2, at the subtelomeric regions of chromosome III, which contain tandem arrays of rDNAs (Fig. 1), sites that are also targeted for heterochromatin formation ${ }^{10}$.

\section{Rik1 loading at heterochromatic repeats}

We have previously suggested that Rik1 may serve to target $\mathrm{Clr} 4$ and the Cul4-dependent ubiquitination machinery to heterochromatic repeats $^{35}$. In support of this model, cells carrying mutant Cul4 (cul4-1) are defective in recruitment of Clr4, but the targeting of Rik1 is not affected ${ }^{35}$. To determine whether Rik1 acts upstream of the loading of other ClrC components at the repeat elements, we examined the localization of different subunits of the complex in
Figure 2 RNAi-dependent loading of Rik1. (a-b) Levels of Flag-Clr4, Flag-Raf1, Raf2-Myc and Rik1-Myc at the mat (cenH) and centromeric (otr $1:$ ura $^{+}$) loci in wild-type and cul4-1 cells were assessed by ChIP. DNA isolated from immunoprecipitated chromatin (ChIP) or whole-cell crude extracts (input) was analyzed by PCR. The intensities of bands representing heterochromatic and control euchromatic loci in ChIP and input lanes were used to calculate the relative fold enrichment values shown below each lane. (c) Mutations of cul4 and c/r3 result in elevated levels of centromeric repeat siRNAs. siRNAs isolated from indicated strains were analyzed by northern blot with a probe specific for $d g$ and $d h$ repeats. (d) Enhanced Rik1 binding at centromeres in cul 4 and c/r3 mutants depends on the RNAi machinery. (e) High-resolution mapping of Rik1 and RNAPII across the silent mat region. A map of the mat locus indicating the PCR fragments amplified in the ChIP assay is shown above. The relative fold enrichments of Rik1-Myc and RNAPII across the silent mat region (see also Supplementary Figs. 3 and 4) are plotted (Rik1, above; RNAPII, below). WT, wild type.

the cul4-1 mutant using ChIP (Fig. 2). The levels of Clr4, Rafl and Raf2 at the cenH element located at the mat locus were reduced in cul4 mutant cells (Fig. 2a), although protein levels of these factors remained unchanged in the cul4- 1 background ${ }^{35}$ (data not shown). In contrast, Rik1 was retained at cenH in cul4 mutant cells, and its levels at this site were in fact higher in mutant cells than in wild-type cells (Fig. 2a). Similar results were obtained when the localization of $\mathrm{ClrC}$ subunits was assayed at a $\mathrm{ura}^{+}$reporter gene inserted at centromeric repeats ( $\operatorname{trl} 1:: u r a 4^{+}$; Fig. 2b). On the basis of these data, Rik1 seems to act upstream of other $\mathrm{ClrC}$ components and could have a role in targeting $\mathrm{ClrC}$ to the heterochromatic repeats.

\section{RNAi-dependent loading of Rik1 at transcribed repeat loci}

RNAPII transcription of heterochromatic repeats is thought to be essential for the generation of siRNAs and for RNAi-mediated targeting of heterochromatin ${ }^{11-14,23}$. cul4-1 cells show upregulated transcription of heterochromatic repeats ${ }^{35}$, correlating with markedly increased siRNA levels (Fig. 2c). We therefore reasoned that the observed increase in Rik1 levels at the repeat loci in these cells might be functionally linked to the RNAi process. Indeed, Rik1 was found delocalized from heterochromatic repeats in cul4 dcrl doublemutant cells (Fig. 2d), suggesting that RNAi processes are involved in Rik1 loading at heterochromatic repeats. To further investigate the role of RNAPII transcription and siRNAs in Rik1 loading, we tested whether deletion of the SHREC subunit Clr3, which participates in heterochromatic transcriptional silencing ${ }^{28}$, also causes elevated Rik1
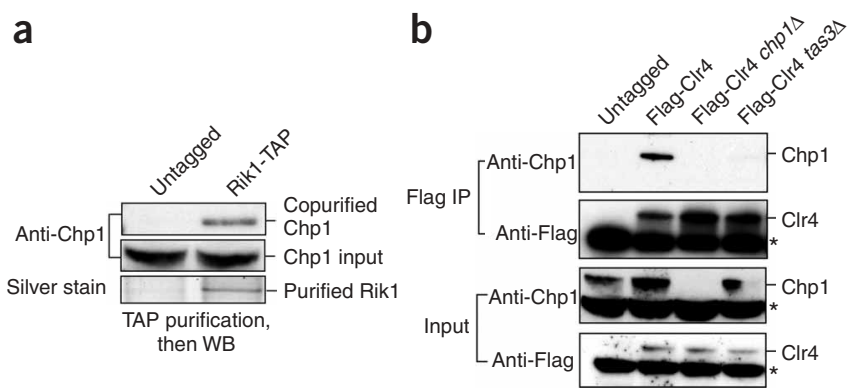

Figure $3 \mathrm{ClrC}$ interacts with RITS. (a) Rik1-TAP purification was followed by silver staining and western blotting using anti-Chp1 antibody. (b) Tas3dependent interaction of Clr4 with Chp1. Immunoprecipitation (IP) using anti-Flag antibody was followed by western blotting using the indicated antibodies. Note a longer exposure time was used to obtain the image shown for the anti-Flag input blot compared to the Flag IP blot. Asterisks indicate nonspecific bands. 

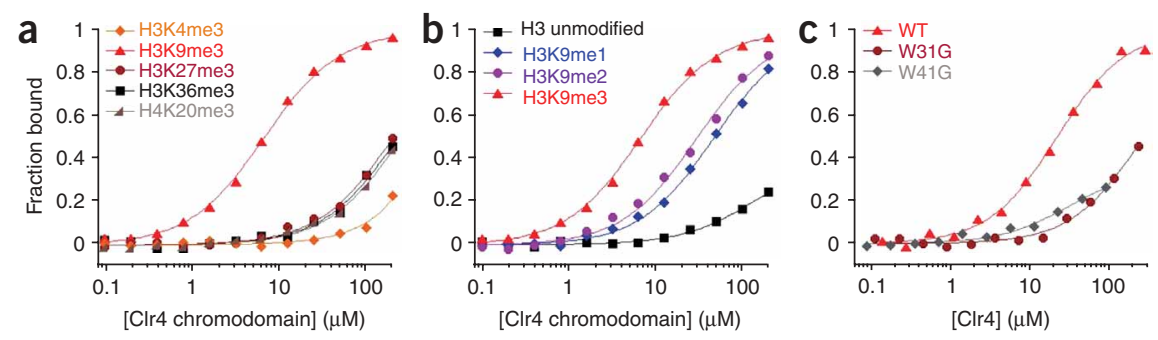

Figure 4 Clr4 chromodomain specifically binds H3K9me in vitro. Fluorescence polarization binding assays of recombinant $\mathrm{Clr} 4$ chromodomain (a,b) or recombinant full-length Clr4 mutant proteins (c) with the indicated fluoresceinated histone peptides. Average results from three experiments are plotted. WT, wild type. and the RdRP activity of Rdp1 are crucial for Rik1 loading, as no Rik1 enrichment could be detected at heterochromatic loci in mutant strains (Supplementary Fig. 5 online). Loss of Rik1 ChIP enrichment at cenH in agol and $r d p 1$ mutants is particularly surprising, as both these factors, unlike Rik1, are dispensable for silencing at the mat locus ${ }^{6,42,43}$. However, as mentioned above, heterochromatin assembly at this locus involves redundant nucleation mechanisms ${ }^{42,43}$. In addition to RNAifacilitated tethering of Rik1 to cenH (this study), DNA binding factors are believed to recruit $\mathrm{ClrC}$ to assemble heterochromatin ${ }^{42}$. levels at repeat loci. Indeed, loss of $\mathrm{Clr} 3$ resulted in increased Rik1 loading at pericentromeric repeats (Fig. 2d). As expected, enhanced Rik1 localization correlated with elevated levels of siRNAs corresponding to $d g$ and $d h$ repeats in $c l r 3$ mutant cells (Fig. 2c). No Rik1 enrichment was detected in $c l r 3 d c r 1$ double-mutant cells (Fig. 2d).

As mentioned above, heterochromatin factors, including ClrC, coat an extended 20-kb domain at the silent mat locus ${ }^{10}$ (Supplementary Fig. 1b). To explore the effects of mutations in cul4 or clr3 on Rik1 localization across the entire silenced domain, we carried out highresolution ChIP with primers distributed across the whole region, as described previously ${ }^{23}$. These experiments showed elevated Rik1 levels specifically at the transcribed cenH element, the RNAi-dependent heterochromatin nucleation center, in both mutants compared to wild-type cells (Fig. 2e and Supplementary Fig. 3 online). Increased Rik1 binding correlated with elevated transcription ${ }^{28,35}$ and RNAPII occupancy at the cenH element in clr3 and cul4 mutants (Fig. 2e and Supplementary Fig. 4 online). Such an increase, however, was not observed in surrounding nontranscribed regions, which in general showed lower Rik1 binding in the mutant background (Fig. 2e). Collectively, these findings suggest that Rik1 is loaded onto heterochromatic repeats in an RNAi-dependent manner, which has important implications for the nucleation of heterochromatic structures.

\section{Rik1 targeting requires Ago1 and Rdp1 catalytic functions}

The RNAi factors Agol and Rdp1 have slicer and RNA-dependent RNA polymerase (RdRP) activities, respectively. These factors are essential for the processing of repeat transcripts into siRNAs ${ }^{14-17}$. We investigated whether the Agol and RdRP enzymatic activities are required for Rik1 targeting to the heterochromatic repeats. For this purpose, we carried out ChIPs using strains carrying mutations in the catalytic site of Agol (ago1 $\left.{ }^{D 580 A}\right)$ or Rdp1 $\left(r d p 1^{D 903 A}\right)$ to investigate Rik1 localization at cenH and the otr1::ura4 ${ }^{+}$reporter gene. Ago ${ }^{\text {D580A }}$ and $\mathrm{Rdp1} 1^{\mathrm{D} 903 \mathrm{~A}}$ mutant proteins are expressed at levels comparable to those of wild-type proteins, but they are defective in their catalytic functions ${ }^{15,17}$. Our experiments showed that the slicer activity of Agol

Figure $5 \mathrm{Clr} 4$ binding to $\mathrm{H} 3 \mathrm{~K} 9$ me facilitates the spreading of $\mathrm{ClrC}$. $(\mathbf{a}, \mathbf{b}) \mathrm{H} 3 \mathrm{~K} 9 \mathrm{R}$ mutation reduces enrichments of $\mathrm{ClrC}$ subunits at the mat and centromeric loci. (c,d) $c / r 4^{W 31 G}$ diminishes $\mathrm{ClrC}$ binding at centromeric repeats and the silent mat region. Levels of Flag-Clr4, Flag-Raf1, Raf2-Myc and Rik1-Myc in the indicated strains were determined by ChIP assay. Relative fold enrichment values are shown below each lane (a-d). (e) Role of Clr4 chromodomain in the spreading of heterochromatin. High-resolution mappings of Raf2-Myc across the silent mat locus in wild-type (WT) or clr4 ${ }^{W 31 G}$ cells were performed using a ChIP assay (see also Supplementary Fig. 7). The relative fold enrichments in the indicated strains are plotted.

\section{ClrC interacts with RITS}

siRNAs bound to Agol are thought to target RITS by base-pairing with nascent repeat transcripts to trigger heterochromatin assembly ${ }^{14,18}$, although the exact mechanism of this process is not known. RNAi-dependent localization of Rik1 and the fact that Rik1 recruitment to repeat loci coincides with RITS loading during $\mathrm{S}$ phase of the cell cycle ${ }^{19}$ prompted us to investigate the possible interactions of these factors. Extracts prepared from cells expressing epitope-tagged Rik1 (Rik1-TAP) were subjected to tandem-affinity purification. Western blot analysis showed that Chp1 (a RITS subunit) copurified with Rik1 but not with fractions purified from untagged control extracts (Fig. 3a). To further confirm these results, we performed coimmunoprecipitation experiments with extracts from cells expressing Flag-tagged Clr4 (Flag-Clr4) and found that Flag-Clr4 interacted with Chp1 (Fig. 3b). This interaction was abolished in cells lacking Tas3 (Fig. 3b), a RITS
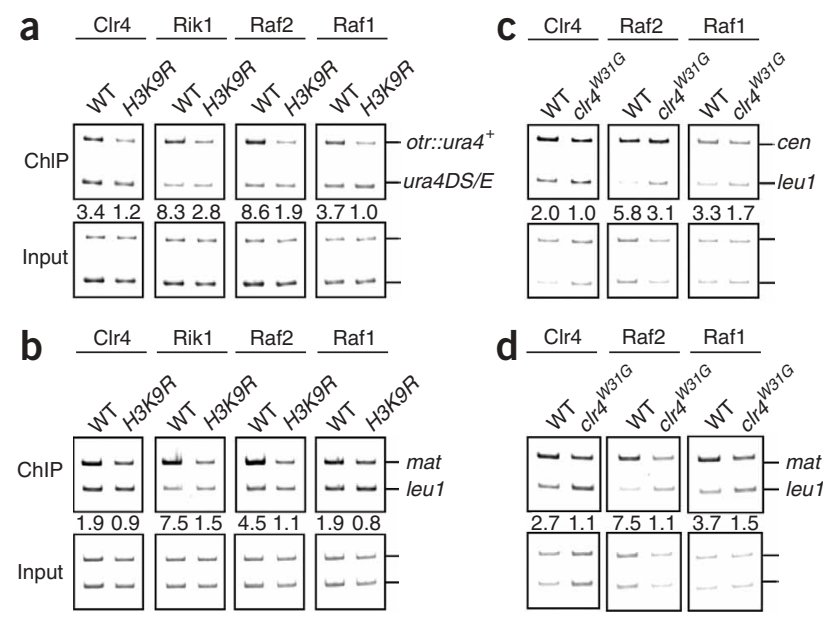

e

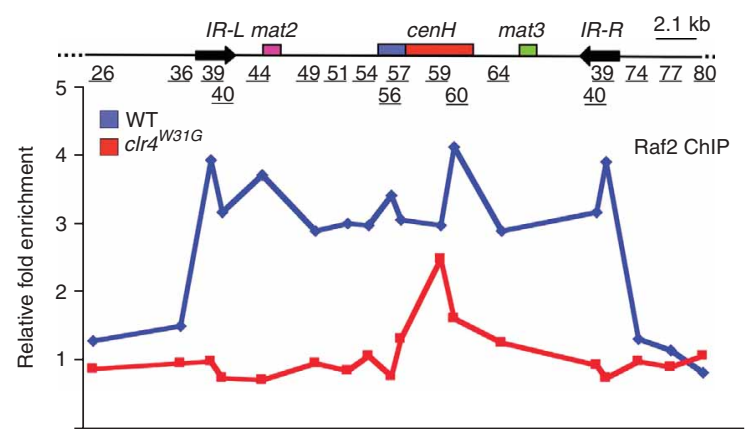


a

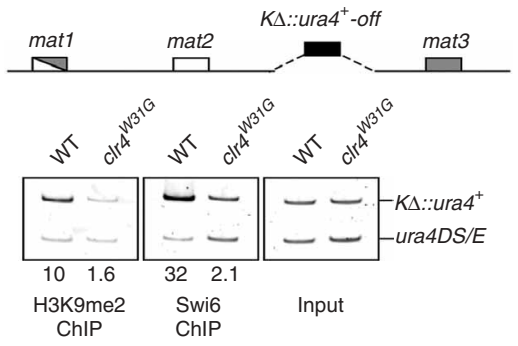

b

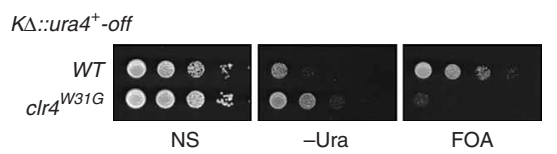

Figure 6 Role of the Clr4 chromodomain in the maintenance of heterochromatin. (a) Mutation of the Clr4 chromodomain results in a reduction in $\mathrm{H} 3 \mathrm{~K} 9 \mathrm{me} 2$ and Swi6 levels at $K \Delta:: \mathrm{ura}^{+}$. A schematic diagram of replacement of the cenH-containing region with $\operatorname{ura}^{+}\left(K \Delta:: u r a 4^{+}\right)$is shown above. (b) A mutation in Clr4 chromodomain adversely affects maintenance of the $K \Delta:: u r a 4^{+}$ura4-off state. Serial dilution plating assays were performed using nonselective (NS), uracil lacking (-Ura) and counterselective 5-fluoroorotic acid (FOA) media to measure $K \Delta:: u r a 4^{+}$expression.

subunit that bridges Chp1 to Agol (ref. 44). Therefore, RITS may directly facilitate $\mathrm{ClrC}$ targeting to heterochromatic repeats.

\section{Clr4 chromodomain binds $\mathrm{H} 3 \mathrm{~K} 9 \mathrm{me}$ in vitro}

Clr4 contains a chromodomain motif ${ }^{32}$ that is essential for efficient methylation of $\mathrm{H} 3 \mathrm{~K} 9$ at heterochromatic loci ${ }^{31}$. However, the precise function of the Clr4 chromodomain in the assembly of heterochromatic structures has not been investigated. We tested whether the chromodomain of Clr4 binds methylated histones in a similar way to the HP1 proteins ${ }^{45}$. Fluorescence polarization binding assays were carried out using recombinant $\mathrm{Clr} 4$ chromodomain and a series of modified histone peptides corresponding to known sites of histone methylation. The Clr4 chromodomain bound specifically to H3K9me but did not bind to H3K4me, H3K27me, H3K36me or H4K20me (Fig. 4a). Much stronger binding was observed for trimethylated H3K9 (H3K9me3), which is preferentially enriched throughout the heterochromatic domains in the S. pombe genome (Supplementary Fig. 6 online), as compared to the mono- or dimethylated counterparts (Fig. $4 \mathrm{~b}$ and Supplementary Table 1 online). Similar findings were obtained for the full-length proteins (Supplementary Table 1). Moreover, phosphorylation of serine $10(\mathrm{H} 3 \mathrm{~S} 10 \mathrm{Ph})$ adjacent to H3K9me3 interfered with the binding of Clr4 to the H3 tail (Supplementary Table 1), which is consistent with a reduction in ClrC levels at heterochromatic regions during mitosis, coincident with increased $\mathrm{H} 3 \mathrm{~S} 10 \mathrm{Ph}$ at these $\operatorname{loci}^{19}$. We also found that recognition of the H3K9me3 mark by Clr4 was severely affected by W31G and W41G mutations (Fig. 4c), which target conserved residues within the aromatic cage of the chromodomain ${ }^{46}$. Together, these data suggest that $\mathrm{Clr} 4$ might not only write but also read the H3K9me mark.

\section{Clr4 binding to $\mathrm{H} 3 \mathrm{~K} 9 \mathrm{me}$ facilitates spreading of $\mathrm{ClrC}$}

The interaction of Clr4 with $\mathrm{H} 3 \mathrm{~K} 9 \mathrm{me}$ could have implications for stable binding of $\mathrm{ClrC}$ at heterochromatic loci. We tested this possibility by performing ChIP using strains expressing a single copy of either wild-type or K9R mutant histone H3 (ref. 47). The H3K9R mutation markedly reduced the localization of $\mathrm{ClrC}$ subunits Clr4, Rik1, Raf1 and Raf2 to the centromeres and the mat locus (Fig. 5a,b). Similarly, we found that the $c l r 4^{W 31 G}$ mutation resulted in diminished enrichment of $\mathrm{ClrC}$ subunits at both centromeres and the mat locus (Fig. $\mathbf{5 c}, \mathbf{d}$ ). Therefore, binding of the $\mathrm{Cl} 4$ chromodomain to $\mathrm{H} 3 \mathrm{~K} 9 \mathrm{me}$ is required for the stable association of $\mathrm{ClrC}$ to heterochromatic loci.

We next tested whether $\mathrm{Clr} 4$ binding to $\mathrm{H} 3 \mathrm{~K} 9 \mathrm{me}$ has a role in the spreading of $\mathrm{ClrC}$ from nucleation sites to surrounding areas. A highresolution map of ClrC subunit Raf2 across the silent mat locus of wild-type and $c l r 4^{W 31 G}$ mutant cells was created using ChIP. Whereas Raf2 was distributed throughout the 20-kb heterochromatic domain in wild-type cells, its localization was mainly restricted to the $\mathrm{cenH}$ element in $c l r 4^{W 31 G}$ mutant cells (Fig. 5e and Supplementary Fig. 7 online). This observation is consistent with the restricted localization of $\mathrm{H} 3 \mathrm{~K} 9 \mathrm{me}$ at the nucleation site in these cells $\mathrm{s}^{23}$. Together, these data indicate that $\mathrm{ClrC}$, which is initially targeted to the cenH element via the RNAi machinery, can spread to adjacent areas through binding of the Clr4 chromodomain to H3K9me.

\section{Heterochromatin maintenance requires $\mathrm{Cl} \mathbf{4}$ chromodomain}

We next investigated whether $\mathrm{Clr} 4$ binding to $\mathrm{H} 3 \mathrm{~K} 9$ me has a role in maintenance of heterochromatin through successive cell divisions. We have shown previously that replacement of the $c e n H$-containing region with $\mathrm{ura}^{+}\left(\mathrm{K \Delta}:: \mathrm{ura}^{+}\right)$results in a metastable locus with alternative silenced (ura4-off) and expressed (ura4-on) epigenetic states, arising from defects in the establishment of heterochromatin ${ }^{7}$. Once established, however, the ura4-off state is remarkably stably inherited in $c i s^{6,7}$. Moreover, the inheritance of the ura4-off state is linked to the maintenance of high levels of $\mathrm{H} 3 \mathrm{~K} 9 \mathrm{me}$ and Swi6 at the locus ${ }^{6,7}$. Our experiments suggest that the cis inheritance of the heterochromatic state in ura4-off cells requires $\mathrm{Cl} 4$ binding to $\mathrm{H} 3 \mathrm{~K} 9 \mathrm{me}$. When we crossed the $K \Delta:: u r a 4^{+}$allele carrying the ura4-off state into the background of the $c l r 4^{W 31 G}$ mutation, the heterochromatic state was lost, as indicated by a severe reduction in $\mathrm{H} 3 \mathrm{~K} 9 \mathrm{me}$ and Swi6 levels at the mat locus (Fig. 6a). Notably, loss of heterochromatin in $c l r 4^{\text {W31G }}$ cells correlated with conversion of the ura4-off to the ura4-on epigenetic state (Fig. 6b). This result suggests the ability of Clr4 to both catalyze H3K9 methylation and bind to H3K9me may provide a mechanism for the inheritance of preassembled heterochromatic structures.

\section{DISCUSSION}

Heterochromatin assembly, which serves as a model for understanding the formation of heritable higher-order chromatin structures, is a complex process that involves at least three distinct phases: initiation, spreading and maintenance. Studies in different experimental systems have identified a conserved set of factors, including histone-modifying enzymes and structural proteins that work together to assemble heterochromatin ${ }^{1,5,8}$. Our genetic and biochemical studies define the contributions of $\mathrm{ClrC}$ subunits in heterochromatin formation across extended chromosomal domains in the S. pombe genome.

Our analyses suggest that Rik1 is a crucial factor involved in RNAimediated targeting of $\mathrm{ClrC}$ to heterochromatic repeat elements. First, we found that, despite a considerable reduction in the localization of the other ClrC subunits in a cul4 mutant, Rik1 levels at heterochromatic repeats were markedly elevated, correlating with enhanced siRNA levels and RNAPII transcription of repeat loci. These findings indicate that loading of Rik 1 is coupled to the RNAPII transcription of centromeric repeat elements. Second, we showed that mutations in RNAi factors that abolish production of siRNAs caused defects in targeting of Rik1 to the transcribed repeat elements, suggesting that RNAi is involved in chromatin loading of Rik1. Third, components of 


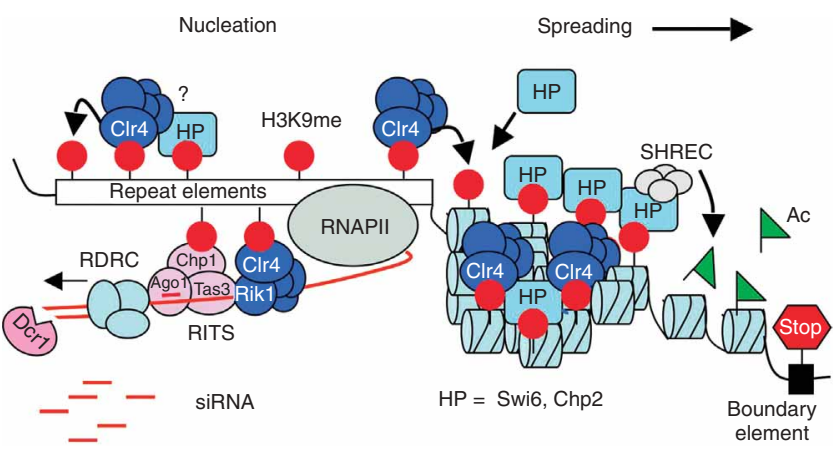

Figure 7 Model showing RNAi-mediated nucleation and spreading of heterochromatin. RNAi factors such as RITS, RDRC and Dicer, involved in processing of repeat transcripts (red line) into siRNAs, are required for targeting of $\mathrm{ClrC}$ to the heterochromatic repeats. siRNA-bound Agol is likely to specify the targeting of RITS to nascent repeat transcripts. RITS then facilitates ClrC loading. Rik1 might also directly associate with the repeat transcript and/or some part of the elongating RNAPII complex, thus promoting ClrC loading to nucleate heterochromatin. After the initial methylation of $\mathrm{H} 3 \mathrm{~K} 9$ by $\mathrm{ClrC}$, $\mathrm{Clr} 4$ bound to $\mathrm{H} 3 \mathrm{~K} 9$ me could modify adjacent nucleosomes creating additional binding sites for $\mathrm{ClrC}$ and other chromodomain proteins including Swi6 and Chp2 (HPs), which in turn mediate recruitment of factors such as SHREC, thereby promoting higher-order chromatin organization. Swi6 could further contribute to long-range heterochromatin spreading by promoting higher-order chromatin organization by forming oligomers and stabilizing the $\mathrm{ClrC}$ binding to chromatin. Boundary DNA elements block inappropriate spreading of heterochromatin into euchromatic regions. Green flag, histone acetylation; red lollipops, H3K9me.

ClrC including Rik1 were found to interact with the RITS complex, which is implicated in siRNA-mediated recruitment of the heterochromatin machinery. These results together with previous findings showing that Rik1 is targeted to heterochromatic repeats coincident with Agol during $S$ phase of the cell cycle ${ }^{19}$ suggest the following model for RNAi-mediated heterochromatin nucleation (Fig. 7). siRNA-bound Agol is likely to provide specificity for targeting RITS to nascent repeat transcripts in a manner similar to the binding of the RNA-induced silencing complex (RISC) RNAi effector to mRNA targets ${ }^{48}$. RITS in turn facilitates ClrC localization. In addition to ClrC interaction with RITS, Rik1 could also directly associate with the repeat transcripts and/or the elongating RNAPII machinery, thus promoting $\mathrm{ClrC}$ localization to nucleate heterochromatin. In this model, components of the RNAPII elongation complex might provide a framework for promoting interaction between ClrC and RITS bound to nascent transcripts via siRNAs. This might occur in a manner analogous to the recruitment of Set1 and Set2 histone methyltransferases by the elongating RNAPII to modify chromatin in the transcribed regions of genes ${ }^{49}$.

Once recruited, $\mathrm{H} 3 \mathrm{~K} 9 \mathrm{me}$ and its associated factors spread along the chromatin fiber beyond the original nucleation site ${ }^{6}$. Although cotranscriptional processes involving RNAi might account for local diffusion of heterochromatin ${ }^{4,16}$, these mechanisms cannot explain long-range spreading of heterochromatin across large chromosomal domains. The results presented here show that binding of Clr4 to $\mathrm{H} 3 \mathrm{~K} 9 \mathrm{me}$ via its chromodomain is crucial for the spreading of heterochromatic structures. Clr4 bound to $\mathrm{H} 3 \mathrm{~K} 9$ me probably modifies adjacent nucleosomes creating additional binding sites for loading of $\mathrm{ClrC}$ and other chromodomain proteins, thus allowing spreading to occur in a step-wise manner (Fig. 7). However, it is unlikely that the Clr4-H3K9me interaction alone is sufficient to bring about the spreading of heterochromatin complexes. As also observed for Swi6 (ref. 20), the binding of Clr4 to H3K9me is weak. As Swi6 has been shown to promote spreading in a dosage-dependent manner ${ }^{6}$, we favor a view where Swi6 bound to H3K9me directly or indirectly stabilizes $\mathrm{ClrC}$ chromatin localization. Indeed, in other systems interaction of Suv39-HP1 (ref. 50-52), besides targeting $\mathrm{H} 3 \mathrm{~K} 9 \mathrm{me}$, is necessary for localization of these factors to heterochromatic loci ${ }^{52}$. In addition to stabilizing Clr4 binding to $\mathrm{H} 3 \mathrm{~K} 9 \mathrm{me}$, Swi6 could further contribute to long-range spreading of heterochromatin by promoting higher-order chromatin organization via oligomerization of its chromoshadow domain ${ }^{53}$. Furthermore, Swi6, along with another chromodomain protein, Chp2, mediates recruitment of factors such as the SHREC chromatin modifier ${ }^{28}$ (B. Cui and S. Grewal, unpublished observations) implicated in spreading of heterochromatin ${ }^{2,28}$.

Given the requirement for $\mathrm{Cl} 4$ binding to $\mathrm{H} 3 \mathrm{~K} 9$ me in maintenance of heterochromatin, a mechanism similar to the one proposed above for spreading might also account for the recapitulation of the specific chromatin configuration following DNA replication. During chromosome replication, the histones originally present are distributed randomly to the daughter chromatids ${ }^{54}$. This potentially leads to 'dilution' of $\mathrm{H} 3 \mathrm{~K} 9$ me nucleosomes in the chromatin as new histones are incorporated. The binding of $\mathrm{Clr} 4$ to $\mathrm{H} 3 \mathrm{~K} 9 \mathrm{me}$ might recruit $\mathrm{ClrC}$ to adjacent newly assembled nucleosomes, thus promoting the re-establishment of the parental histone-modification pattern as well as clonal propagation of heterochromatic structures in cis. This process is probably also facilitated by Swi6 or HP1 proteins that interact both with histone modifiers ${ }^{50,51}$ and with factors involved in replication-coupled chromatin assembly, such as chromatin assembly factor $1(\mathrm{CAF} 1)^{55,56}$. Other histone chaperones such as histone regulator A (HIRA), implicated in heterochromatic silencing ${ }^{57}$, are also likely to be involved in this process. Regardless of the detailed mechanism, we note that the ability of Clr4 to both write and read the $\mathrm{H} 3 \mathrm{~K} 9 \mathrm{me}$ mark underlies the propagation of heterochromatic structures.

Considering that Clr4 (Suv39h) and Swi6 (HP1) proteins are conserved in multicellular organisms, a similar mechanism may also operate in other species. In this regard, we note that Suv39h also contains both writing (SET domain) and reading (chromodomain) modules $^{58}$. Indeed, H3K9 methyltransferases that regulate expression of specific loci but lack a chromodomain seem incapable of mediating heterochromatin spreading 45,58 . Therefore, our results showing a role for the $\mathrm{Cl} 4-\mathrm{H} 3 \mathrm{~K} 9 \mathrm{me}$ interaction in the spreading of heterochromatin may have implications for understanding the basis for genespecific versus long-range effects of heterochromatin in different chromosomal contexts.

Future investigations into the assembly and maintenance of heterochromatin in $S$. pombe are expected to have wide-ranging implications for our understanding of chromatin-directed and noncoding RNAdirected epigenetic mechanisms in the developmental regulation of gene expression and other chromosomal processes. In this regard, we note that Cul4-based ubiquitin E3 ligases, which regulate various cellular functions such as DNA replication and the DNA damage response, form complexes with multiple WD domain proteins essential for histone methylation and epigenetic gene regulation in higher eukaryotes ${ }^{59}$.

\section{METHODS}

Strain constructions. Raf2- $(13 \times)$ Myc strain was constructed with a PCR-based method. For generating $(3 \times)$ Flag-Rafl strain, the KanMX marker gene was first integrated at the $3^{\prime}$ UTR region of rafi $1^{+}$. We cloned a fragment containing the 
raf1 gene with KanMX integrated at its $3^{\prime}$ UTR into the pCR-Blunt II-TOPO vector (Invitrogen) to generate pCR-TOPO-rafl-C-KanMX. A $(3 \times)$ Flag-tag sequence was inserted at the $5^{\prime}$ end of the raf1 gene of pCR-TOPO-raf1C-KanMX plasmid to create pCR-TOPO-3X Flag-raf1-C-KanMX. A strain expressing functional Flag-Rafl was constructed by transforming the $(3 \times)$ Flag-raf1-C-KanMX cassette into a wild-type strain, followed by selection for G418 positive colonies, which were subjected to DNA sequencing to confirm proper insertion of $(3 \times)$ Flag-raf1. $(3 \times)$ Flag-Clr4, $(3 \times)$ Flag-Cul4 and Rikl-(13×)Myc strains used in this study were described previously ${ }^{35}$. We used standard genetic crosses to construct all other strains.

Chromatin immunoprecipitation and ChIP-chip. ChIP and ChIP-chip analyses were performed as described previously ${ }^{10}$ using antibodies against Swi6 (ref. 7); di- or tri-methylated H3K9 (Upstate); Flag (M2, Sigma); Myc (Covance and Santa Cruz); RNAPII (8WG16, Covance). ChIP-chip data are available at the US National Center for Biotechnology Information Gene Expression Omnibus (NCBI GEO) under the accession number GSE10561.

Binding assays. We carried out binding assays with recombinant His-Clr4 chromodomain or full-length His-Clr4 and GST-Clr4 proteins purified by affinity chromatography and gel filtration using procedures described previously ${ }^{60}$. The peptides used were: $\mathrm{H} 3{ }^{(1-15)} \mathrm{H}_{2} \mathrm{~N}-A R T K Q T A R K S T G G K A Y-$ $\mathrm{COOH}$ with unmodified $\mathrm{K}$, or modified $\mathrm{K} 9 \mathrm{me} 1, \mathrm{~K} 9 \mathrm{me} 2$ and $\mathrm{K} 9 \mathrm{me}$; $\mathrm{H} 3$ ${ }^{(19-35)} \mathrm{H}_{2} \mathrm{~N}$-QLATKAARKSAPATGGVY-COOH K27me3; H3 ${ }^{(28-43)} \mathrm{H}_{2} \mathrm{~N}$-SAPA TGGVKKPHRYRPY-COOH K36me3; and $\mathrm{H}_{4}{ }^{(12-27)} \mathrm{H}_{2} \mathrm{~N}-\mathrm{KGGAKRHRKVLR}$ DNIQ-COOH K20me3. The identity of all peptides was verified by MALDI-MS.

Protein purification and immunoprecipitation. For Rik1 purification, cells harvested from 2-liter cultures (optical density at $595 \mathrm{~nm}\left(\mathrm{OD}^{595}\right)=3.5$ ) were suspended in $\mathrm{K}$ buffer $(20 \mathrm{mM}$ HEPES at $\mathrm{pH} 7.4,350 \mathrm{mM} \mathrm{KCl}, 0.1 \%(\mathrm{v} / \mathrm{v})$ Tween-20, $1 \mathrm{mM}$ DTT) containing complete protease inhibitors (Roche) and PMSF $(1 \mathrm{mM})$. All subsequent steps were performed at $4{ }^{\circ} \mathrm{C}$. Cells were ground with $40 \mathrm{~g}$ of glass beads using a Puluerisette 6 system. The program was set up as power 500, $4 \mathrm{~min}$, two repeats with $1 \mathrm{~min}$ rest interval. Beads and lysate was transferred into a $50-\mathrm{ml}$ syringe and pressed into Falcon tubes. Cell debris was removed by centrifugation at $3,000 \times g$ for $10 \mathrm{~min}$, and lysate was cleared by centrifugation at $27,000 \times g$ for $1 \mathrm{~h}$. The cleared lysate was incubated with $200 \mu \mathrm{lgG}$ Sepharose (Amersham) for $2 \mathrm{~h}$ at $4{ }^{\circ} \mathrm{C}$. Beads were washed by gravity flow-through with $10 \mathrm{ml} \mathrm{K}$ buffer, followed by $10 \mathrm{ml} \mathrm{K}$ buffer containing $500 \mathrm{mM} \mathrm{KCl}$. Bound proteins were eluted from the beads by rocking overnight in $300 \mu \mathrm{K}$ buffer containing $4 \mu \mathrm{g}$ TEV protease (Invitrogen). The TEV eluate was precipitated by trichloroacetic acid (TCA), and the protein pellet was dried and resuspended in $50 \mu 11 \times$ SDS loading buffer. The Flag-Clr4 immunoprecipitation was performed as described ${ }^{35}$ with minor modifications. Cells harvested from 2 -liter cultures $\left(\mathrm{OD}^{595}=3.5\right)$ were suspended in $2 \times \mathrm{HC}$ buffer (300 mM HEPES at pH 7.4, $100 \mathrm{mM} \mathrm{KCl,} 2 \mathrm{mM}$ EDTA, $0.2 \%$ $(\mathrm{v} / \mathrm{v})$ Nonidet P-40, $2 \mathrm{mM}$ DTT) containing complete protease inhibitors (Roche) and PMSF. Cell extracts were made by grinding $25 \mathrm{ml}$ of suspended cells with $40 \mathrm{~g}$ glass beads using a Puluerisette 6 system. The cleared lysate was incubated with $100 \mu \mathrm{l}$ anti-Flag agarose (M2, Sigma) for $2 \mathrm{~h}$ at $4{ }^{\circ} \mathrm{C}$. Beads were washed by gravity flow-through with $10 \mathrm{ml} 1 \times$ HC buffer $(300 \mathrm{mM}$ HEPES at pH 7.4, $250 \mathrm{mM} \mathrm{KCl}, 1 \mathrm{mM}$ EDTA, $0.1 \%$ (v/v) Nonidet P- $40,1 \mathrm{mM}$ DTT), followed by $10 \mathrm{ml} \mathrm{AC}$ buffer (20 mM HEPES at pH 7.4, $250 \mathrm{mM} \mathrm{KCl}$, $2 \mathrm{mM} \mathrm{MgCl}_{2}, 1 \mathrm{mM}$ EGTA, $0.1 \%$ (v/v) Nonidet P-40, $1 \mathrm{mM} \mathrm{DTT}$ ). Bound proteins were eluted twice from the beads by rocking for $1 \mathrm{~h}$ in $150 \mu \mathrm{l} \mathrm{AC}$ buffer containing $300 \mu \mathrm{g} \mathrm{ml}^{-1}$ of Flag peptide (Sigma). Immunoprecipitated fractions and the equivalent of $5 \%$ of the input extracts were analyzed by western blot using anti-Chp1 (Abcam) and anti-Flag (M2, Sigma) antibodies.

RNA analysis. We carried out northern blot analysis for detecting siRNAs as previously described ${ }^{28}$.

Note: Supplementary information is available on the Nature Structural \& Molecular Biology website.

\section{ACKNOWLEDGMENTS}

We thank C. Peterson (University of Massachusetts Medical School, Worcester, MA, USA) and R. Allshire (Wellcome Trust Center for Cell Biology, Edinburgh) for strains, members of the Grewal laboratory for helpful discussions, and S. Jia and C. Denby for strain constructions. We also thank H. Cam for editing the manuscript. This research was supported by the Intramural Research Program of the National Institutes of Health, National Cancer Institute.

Published online at http://www.nature.com/nsmb/

Reprints and permissions information is available online at http://npg.nature.com/ reprintsandpermissions

1. Ebert, A., Lein, S., Schotta, G. \& Reuter, G. Histone modification and the control of heterochromatic gene silencing in Drosophila. Chromosome Res. 14, 377-392 (2006).

2. Grewal, S.I. \& Jia, S. Heterochromatin revisited. Nat. Rev. Genet. 8, 35-46 (2007).

3. Lamb, J.C., Yu, W., Han, F. \& Birchler, J.A. Plant chromosomes from end to end: telomeres, heterochromatin and centromeres. Curr. Opin. Plant Biol. 10, 116-122 (2007).

4. Talbert, P.B. \& Henikoff, S. Spreading of silent chromatin: inaction at a distance. Nat. Rev. Genet. 7, 793-803 (2006).

5. Grewal, S.I. \& Elgin, S.C. Heterochromatin: new possibilities for the inheritance of structure. Curr. Opin. Genet. Dev. 12, 178-187 (2002).

6. Hall, I.M. et al. Establishment and maintenance of a heterochromatin domain. Science 297, 2232-2237 (2002)

7. Nakayama, J., Klar, A.J. \& Grewal, S.I. A chromodomain protein, Swi6, performs imprinting functions in fission yeast during mitosis and meiosis. Cell 101, 307-317 (2000).

8. Wallace, J.A. \& Orr-Weaver, T.L. Replication of heterochromatin: insights into mechan isms of epigenetic inheritance. Chromosoma 114, 389-402 (2005).

9. Gregory, R.I. \& Shiekhattar, R. Chromatin modifiers and carcinogenesis. Trends Cell Biol. 14, 695-702 (2004).

10. Cam, H.P. et al. Comprehensive analysis of heterochromatin- and RNAi-mediated epigenetic control of the fission yeast genome. Nat. Genet. 37, 809-819 (2005).

11. Volpe, T.A. et al. Regulation of heterochromatic silencing and histone H3 lysine-9 methylation by RNAi. Science 297, 1833-1837 (2002).

12. Djupedal, I. et al. RNA Pol II subunit Rpb7 promotes centromeric transcription and RNAi-directed chromatin silencing. Genes Dev. 19, 2301-2306 (2005).

13. Kato, H. et al. RNA polymerase II is required for RNAi-dependent heterochromatin assembly. Science 309, 467-469 (2005).

14. Motamedi, M.R. et al. Two RNAi complexes, RITS and RDRC, physically interact and localize to noncoding centromeric RNAs. Cell 119, 789-802 (2004).

15. Sugiyama, T., Cam, H., Verdel, A., Moazed, D. \& Grewal, S.I. RNA-dependent RNA polymerase is an essential component of a self-enforcing loop coupling heterochromatin assembly to siRNA production. Proc. Natl. Acad. Sci. USA 102, 152-157 (2005).

16. Irvine, D.V. et al. Argonaute slicing is required for heterochromatic silencing and spreading. Science 313, 1134-1137 (2006).

17. Zofall, M. \& Grewal, S.I. RNAi-mediated heterochromatin assembly in fission yeast. Cold Spring Harb. Symp. Quant. Biol. 71, 487-496 (2006).

18. Verdel, A. et al. RNAi-mediated targeting of heterochromatin by the RITS complex. Science 303, 672-676 (2004)

19. Chen, E.S. et al. Cell cycle control of centromeric repeat transcription and heterochromatin assembly. Nature 451, 734-737 (2008).

20. Bannister, A.J. et al. Selective recognition of methylated lysine 9 on histone $\mathrm{H} 3$ by the HP1 chromo domain. Nature 410, 120-124 (2001).

21. Nakayama, Ji, Allshire, R.C., Klar, A.J. \& Grewal, S.I. A role for DNA polymerase $\alpha$ in epigenetic control of transcriptional silencing in fission yeast. EMBO J. 20, 2857-2866 (2001).

22. Sadaie, M., lida, T., Urano, T. \& Nakayama, J. A chromodomain protein, Chp1, is required for the establishment of heterochromatin in fission yeast. EMBO J. 23, 3825-3835 (2004).

23. Noma, K. et al. RITS acts in cis to promote RNA interference-mediated transcriptional and post-transcriptional silencing. Nat. Genet. 36, 1174-1180 (2004).

24. Kanoh, J., Sadaie, M., Urano, T. \& Ishikawa, F. Telomere binding protein Taz1 establishes Swi6 heterochromatin independently of RNAi at telomeres. Curr. Biol. 15, 1808-1819 (2005).

25. Shankaranarayana, G.D., Motamedi, M.R., Moazed, D. \& Grewal, S.I. Sir2 regulates histone H3 lysine 9 methylation and heterochromatin assembly in fission yeast. Curr. Biol. 13, 1240-1246 (2003).

26. Freeman-Cook, L.L. et al. Conserved locus-specific silencing functions of Schizosaccharomyces pombe sir2 ${ }^{+}$. Genetics 169, 1243-1260 (2005).

27. Nicolas, E. et al. Distinct roles of HDAC complexes in promoter silencing, antisense suppression and DNA damage protection. Nat. Struct. Mol. Biol. 14, 372-380 (2007).

28. Sugiyama, T. et al. SHREC, an effector complex for heterochromatic transcriptiona silencing. Cell 128, 491-504 (2007).

29. Wiren, M. et al. Genomewide analysis of nucleosome density histone acetylation and HDAC function in fission yeast. EMBO J. 24, 2906-2918 (2005).

30. Rea, S. et al. Regulation of chromatin structure by site-specific histone $\mathrm{H} 3$ methyltransferases. Nature 406, 593-599 (2000).

31. Nakayama, J., Rice, J.C., Strahl, B.D., Allis, C.D. \& Grewal, S.I. Role of histone H3 Iysine 9 methylation in epigenetic control of heterochromatin assembly. Science $\mathbf{2 9 2}$ 110-113 (2001).

32. Ivanova, A.V., Bonaduce, M.J., Ivanov, S.V. \& Klar, A.J. The chromo and SET domains of the CIr4 protein are essential for silencing in fission yeast. Nat. Genet. 19, 192-195 (1998). 
33. Horn, P.J., Bastie, J.N. \& Peterson, C.L.A. Rik1-associated, cullin-dependent E3 ubiquitin ligase is essential for heterochromatin formation. Genes Dev. 19, 1705-1714 (2005).

34. Hong, E.J., Villen, J., Gerace, E.L., Gygi, S.P. \& Moazed, D. A cullin E3 ubiquitin ligase complex associates with Rik1 and the Clr4 histone H3-K9 methyltransferase and is required for RNAi-mediated heterochromatin formation. RNA Biol. 2, 106-111 (2005).

35. Jia, S., Kobayashi, R. \& Grewal, S.I. Ubiquitin ligase component Cul4 associates with Clr4 histone methyltransferase to assemble heterochromatin. Nat. Cell Biol. 7, 1007-1013 (2005).

36. Thon, G. et al. The CIr7 and Clr8 directionality factors and the Pcu4 cullin mediate heterochromatin formation in the fission yeast Schizosaccharomyces pombe. Genetics 171, 1583-1595 (2005).

37. Neuwald, A.F. \& Poleksic, A. PSI-BLAST searches using hidden Markov models of structural repeats: prediction of an unusual sliding DNA clamp and of $\beta$-propellers in UV-damaged DNA-binding protein. Nucleic Acids Res. 28, 3570-3580 (2000).

38. Li, F. et al. Two novel proteins, Dos1 and Dos2, interact with Rik1 to regulate heterochromatic RNA interference and histone modification. Curr. Biol. 15, 1448-1457 (2005).

39. Greil, F. et al. Distinct HP1 and Su(var)3-9 complexes bind to sets of developmentally coexpressed genes depending on chromosomal location. Genes Dev. 17, 2825-2838 (2003).

40. Nielsen, S.J. et al. Rb targets histone $\mathrm{H} 3$ methylation and $\mathrm{HP} 1$ to promoters. Nature 412, 561-565 (2001).

41. Holmberg, C. et al. Ddb1 controls genome stability and meiosis in fission yeast. Genes Dev. 19, 853-862 (2005).

42. Jia, S., Noma, K. \& Grewal, S.I. RNAi-independent heterochromatin nucleation by the stress-activated ATF/CREB family proteins. Science 304, 1971-1976 (2004).

43. Yamada, T., Fischle, W., Sugiyama, T., Allis, C.D. \& Grewal, S.I. The nucleation and maintenance of heterochromatin by a histone deacetylase in fission yeast. Mol. Cell 20, 173-185 (2005).

44. Partridge, J.F. et al. Functional separation of the requirements for establishment and maintenance of centromeric heterochromatin. Mol. Cell 26, 593-602 (2007).

45. Martin, C. \& Zhang, Y. The diverse functions of histone lysine methylation. Nat. Rev. Mol. Cell Biol. 6, 838-849 (2005).
46. Jacobs, S.A. \& Khorasanizadeh, S. Structure of HP1 chromodomain bound to a lysine 9-methylated histone H3 tail. Science 295, 2080-2083 (2002).

47. Mellone, B.G. et al. Centromere silencing and function in fission yeast is governed by the amino terminus of histone H3. Curr. Biol. 13, 1748-1757 (2003).

48. Filipowicz, W. RNAi: the nuts and bolts of the RISC machine. Cell 122, 17-20 (2005).

49. Hampsey, M. \& Reinberg, D. Tails of intrigue: phosphorylation of RNA polymerase II mediates histone methylation. Cell 113, 429-432 (2003)

50. Aagaard, L. et al. Functional mammalian homologues of the Drosophila PEV-modifier Su(var)3-9 encode centromere-associated proteins which complex with the heterochromatin component M31. EMBO J. 18, 1923-1938 (1999).

51. Schotta, G. et al. Central role of Drosophila SU(VAR)3-9 in histone H3-K9 methylation and heterochromatic gene silencing. EMBO J. 21, 1121-1131 (2002).

52. Stewart, M.D., Li, J. \& Wong, J. Relationship between histone H3 lysine 9 methylation, transcription repression, and heterochromatin protein 1 recruitment. Mol. Cell. Biol. 25, 2525-2538 (2005)

53. Cowieson, N.P., Partridge, J.F., Allshire, R.C. \& McLaughlin, P.J. Dimerisation of a chromo shadow domain and distinctions from the chromodomain as revealed by structural analysis. Curr. Biol. 10, 517-525 (2000).

54. Jackson, V. \& Chalkley, R. Histone segregation on replicating chromatin. Biochemistry 24, 6930-6938 (1985).

55. Murzina, N., Verreault, A., Laue, E. \& Stillman, B. Heterochromatin dynamics in mouse cells: interaction between chromatin assembly factor 1 and HP1 proteins. Mol. Cell 4, 529-540 (1999).

56. Quivy, J.P. et al. A CAF-1 dependent pool of HP1 during heterochromatin duplication. EMBO J. 23, 3516-3526 (2004).

57. Greenall, A. et al. Hip3 interacts with the HIRA proteins Hip1 and SIm9 and is required for transcriptional silencing and accurate chromosome segregation. J. Biol. Chem. 281, 8732-8739 (2006).

58. Kouzarides, T. Histone methylation in transcriptional control. Curr. Opin. Genet. Dev. 12, 198-209 (2002).

59. Higa, L.A. et al. CUL4-DDB1 ubiquitin ligase interacts with multiple WD40-repeat proteins and regulates histone methylation. Nat. Cell Biol. 8, 1277-1283 (2006).

60. Fischle, W. et al. Regulation of HP1-chromatin binding by histone $\mathrm{H} 3$ methylation and phosphorylation. Nature 438, 1116-1122 (2005). 